\title{
Classes de comprimento e proporção sexual em Diplodon expansus (Mollusca, Bivalvia, Hyriidae) no rio Piraquara, Paraná, Brasil
}

\author{
Ana A. N. Meyer, Edinalva Oliveira \& Jessica Martim
}

Universidade Positivo, Rua Prof. Pedro Viriato Parigot de Souza, 5300, 81280-330 Campo Comprido, Curitiba, Paraná, Brasil. (anameyer@onda.com.br; edinaoli@yahoo.com.br; jessica.martim@gmail.com)

\begin{abstract}
Length classes and sex ratio in Diplodon expansus (Mollusca, Bivalvia, Hyridae) in the Piraquara river, Paraná, Brazil. This study aimed to analyze the distribution of size classes and sex ratio to broaden the knowledge on the reproductive behavior of Diplodon expansus (Küster, 1856) and the biodiversity of the water bodies of the Área de Proteção Ambiental Piraquara, Paraná, Brazil. The study area is a section of the Piraquara river, characterized by lotic water and relatively well preserved riparian vegetation. Four samplings were carried out from March 2006 to December 2006. In the Piraquara river, D. expansus occurs in sections of the river with sandy and slimy substrate. The length of specimens ranged from $17 \mathrm{~mm}$ to $65 \mathrm{~mm}$, and the highest frequencies of length classes were found for intermediate sizes. Histological analysis of gonads revealed a 1:1 sex ratio. No hermaphrodite specimens were found, characterizing a dioecious population. Qualitative and quantitative analysis showed a continuous gametogenesis, with peaks of larval release in the summer.
\end{abstract}

KEYWORDS. Unionoida, reproduction, gametogenesis, histology.

RESUMO. O presente estudo tem como objetivo analisar a distribuição de classes de tamanho e proporção sexual ampliando o conhecimento do comportamento reprodutivo de Diplodon expansus (Küster, 1856) e da biodiversidade dos corpos de água da Área de Proteção Ambiental do Piraquara, Paraná, Brasil. A área de estudo corresponde a um trecho do rio Piraquara de águas lóticas, e mata ciliar relativamente bem preservada. Foram realizadas quatro coletas no período de março de 2006 a dezembro de 2006 . No rio Piraquara o $D$. expansus ocorre em substratos arenoso e lodoso. O comprimento dos exemplares variou entre $17 \mathrm{~mm}$ e $65 \mathrm{~mm}$, sendo que a maior frequência de classes de comprimento foi registrada para as classes intermediárias. A análise histológica das gônadas possibilitou a determinação de uma razão sexual 1:1, não tendo sido identificado nenhum exemplar hermafrodita, caracterizando uma população tipicamente dióica. Análises qualitativas e quantitativas demonstram uma gametogênese contínua, com picos de liberação larval no verão.

PALAVRAS-CHAVE. Unionoida, reprodução, gametogênese, histologia.

Os bivalves de água doce possuem grande importância ecológica. Por serem organismos filtradores, controlam a quantidade de fitoplâncton, detritos e partículas inorgânicas, promovendo a diminuição da turgidez e o aumento da penetração de luz para macrófitas submersas, das quais uma variedade de outros animais depende (VAughn \& HaKenKamp, 2001). No Brasil, gêneros das quatro famílias de bivalves límnicos (Mycetopodidae, Hyriidae, Sphaeriidae e Corbiculidae) possuem representantes na lista da fauna ameaçada de extinção, sendo o maior número de espécies registradas para os estados do sul e sudeste, que pode ser entre outros fatores, reflexo do maior nível de desenvolvimento econômico que resulta em maior impacto ambiental para os sistemas aquáticos (AgostinHo et al., 2005).

Segundo Boneto (1961), o gênero Diplodon Spix, 1827 possui larga distribuição na America do Sul. No Chile, a espécie Diplodon chilensis (Gray, 1828) é considerada a mais abundante em termos de biomassa (PARADA et al., 2007), e trabalhos sobre a organização gonadal e gametogênese (PEREdo \& PArAdA, 1984), distribuição espacial e densidade (LARA \& PARADA, 1988), crescimento e ciclo de vida (PARADA, et al, 1989), táticas reprodutivas e dinâmica populacional (PARADA et al., 1990), características populacionais e ciclo de vida (Semenas \& Brugni, 2002), possibilitam um amplo entendimento sobre a espécie. Para a Argentina os trabalhos com bivalves límnicos possuem como principal enfoque a taxonomia e distribuição geográfica (PARODIZ \& BONETTO, 1963).
No território brasileiro, dez espécies do gênero Diplodon, entre elas Diplodon expansus (Küster, 1856) possuem status de espécie ameaçada, principalmente devido à poluição dos rios, desmatamento e competição com espécies exóticas (MMA, 2008). Características do gênero para o Brasil estão restritas a estudos da morfologia do sistema digestivo (MANSUR, 1973), revisão taxonômica (MANSur \& Pereira, 2006) e morfologia de gloquídios (MANSUR, 1999) realizados para o Rio Grande do Sul. AvelAR \& MendonçA (1998) descreveram aspectos da gametogênese de Diplodon rotundus gratus (Wagner, 1827), HENRY \& SiMÃo (1984) a avaliação da densidade e biomassa de Diplodon delontus expansus e Avelar \& CunHa (2009) a anatomia funcional de Diplodon rhombeus fontainianus (Orbigny, 1835) para o estado de São Paulo.

O estado do Paraná apresenta uma grande área de águas continentais, constituídas por rios, lagos e represas. Em contraste com a dimensão destes sistemas límnicos, a biodiversidade é ainda pouco conhecida e os estudos de espécies nativas de bivalves estão restritos aos realizados por CURIAL \& LANGE (1974a, b; 1975), que analisaram a proporção sexual e relatos de hermafroditismo para $D$. delontus expansus no rio Cerne, um afluente do rio Capivari, pertencente à bacia hidrográfica do rio Ribeira, em trecho localizado na região metropolitana de Curitiba, PR.

O presente estudo tem como objetivo analisar a distribuição de classes de tamanho e a proporção sexual em $D$. expansus, ampliando o conhecimento sobre o seu comportamento reprodutivo e sobre a biodiversidade límnica da Área de Proteção Ambiental do Piraquara. 


\section{MATERIAL E MÉTODOS}

Área de estudo. A bacia hidrográfica do rio Piraquara está localizada no município de Piraquara (Região Metropolitana de Curitiba, PR) e pertence à bacia hidrográfica do Altíssimo Iguaçu. As águas do rio Piraquara são regularizadas através de barragem existente, cuja bacia possui $27 \mathrm{~km}^{2}$ de área, sendo protegida através de Decreto Estadual no 1754 de 06/05/96 que criou a APA do rio Piraquara.

A flora predominante é constituída de Floresta Ombrófila Mista (MAACK, 1981). O clima segundo o sistema de classificação de Koppen é Cfb, com verões frescos, inverno com temperaturas baixas e ausência de estação seca (MARQUES et al., 2003).

A área de estudo compreende o trecho do rio Piraquara localizado a montante da represa de Piraquara I e II ( $25^{\circ} 30^{\prime} \mathrm{S}, 49^{\circ} 00^{\prime} \mathrm{W}$; altitude $\left.916 \mathrm{~m}\right)$, apresentando uma largura média de $4,50 \mathrm{~m}$ e profundidade variando entre $30 \mathrm{~cm}$ nas margens a $70 \mathrm{~cm}$ na região central do leito do rio. $\mathrm{O}$ fundo apresenta trechos lodosos entremeados por área de areia, com diferente granulometria e rochas de variados portes.

Os exemplares D. expansus utilizados no presente estudo foram coletados sob licença 0217.001078/2006-91 concedida pelo Instituto Brasileiro do Meio Ambiente e dos Recursos Renováveis em quatro coletas sazonais, realizadas ao final de cada estação climática no período entre março de 2006 a dezembro de 2006. Os dados abióticos foram mensurados em campo. A temperatura do ar e da água foram obtidas através de termômetro de mercúrio, a condutividade através de condutivímetro portátil e o pH com papel de tornassol. Para obtenção da velocidade de corrente foi determinado uma seção com $10 \mathrm{~m}$. Na marca zero metros foi colocada uma bola de isopor e cronometrado o tempo gasto para deslocamento até a marca $10 \mathrm{~m}$. O procedimento foi realizado na margem direita e esquerda e na região central do leito do rio. Os dados resultantes foram aplicados na fórmula $\mathrm{V}=\mathrm{d} / \mathrm{t}$ e a velocidade de corrente final obtida através da média das velocidades aferidas.

Os bivalves foram coletados com auxílio de peneiras de $50 \mathrm{~cm}$ de diâmetro e malha de $1 \mathrm{~mm}$, inseridas em movimento de varredura no leito do rio. Os primeiros 50 exemplares coletados de cada uma das quatro amostragens foram acondicionados em tanques de transporte contendo água do local de coleta e aeração constante. Em laboratório foi realizada abertura mecânica das valvas e fixação em formol $10 \%$ por $48 \mathrm{~h}$. Para a determinação da frequência de classes de tamanho, de cada exemplar foi obtido o comprimento total da concha, segundo a metodologia descrita por MANsur et al. (1987), utilizando-se paquímetro de legibilidade $0,05 \mathrm{~mm}$. Os exemplares foram identificados e examinados através de lupa para determinação da presença de marsúpio nas demibrânquias. Cada exemplar recebeu identificação numérica, as partes moles foram conservadas em álcool $70 \%$ e as conchas arquivadas em lotes em seco. Lotes de material testemunho, constituídos de conchas e exemplares com partes moles, foram depositados na Coleção de Mollusca do Museu de História Natural Capão da Imbuia, MHNCI 4774-4778.

Para a determinação histológica do sexo e análises quantitativas da gametogênese, de 186 exemplares, foram obtidas secções transversais da região central da massa visceral, as quais foram submetidas à desidratação, diafanização e inclusão em parafina. Cortes seriados de 5 $\mu \mathrm{m}$ foram corados com Hematoxilina e Eosina e Alcian Blue $\mathrm{pH}$ 2,5. A gametogênese feminina foi quantificada através da medida média de ovócitos e do número de ovócitos por folículo gonadal em metodologia similar a utilizada por JoNEs et al. (1986). Em cada secção foram medidos 30 ovócitos, cujo plano de corte permitia a visualização do núcleo celular. As medidas foram obtidas através de microscópio óptico e software Image Proplus ${ }^{\circledR}$. De cada ovócito foram obtidas duas medidas, uma ao longo do maior eixo e a segunda no eixo perpendicular incidindo no ponto médio do primeiro eixo. A média das duas medidas foi utilizada para determinação do diâmetro do ovócito. Em cada secção transversal foram contados os ovócitos presentes na luz de 30 folículos gonadais. Os dados temporais da gametogênese feminina foram analisados através da ANOVA, com resultados sendo considerados significativos somente se $p<0,05$. O intervalo de amplitude das classes de tamanho foi determinado a partir da aplicação da Regra de Sturgers e a proporção sexual amostrada através de teste de quiquadrado.

\section{RESULTADOS}

$\mathrm{Na}$ área de captura do D. expansus, o rio Piraquara apresenta substrato lodoso, com acúmulo de matéria orgânica vegetal nas porções marginais, cuja profundidade máxima observada nos períodos de coleta foi de $30 \mathrm{~cm}$. Na região central do leito do rio o substrato é arenoso, com profundidade máxima observada de 70 $\mathrm{cm}$. Os dados abióticos em cada coleta estão sumariados na Tabela I.

Após as triagens 14 exemplares não apresentaram forma de conchas característica de $D$. expansus, tendo sido excluídos das análises. Foram registradas nove classes de comprimento com amplitude de intervalo de 6 $\mathrm{mm}(\mathrm{n}=186)$ (Fig. 1). O comprimento mínimo observado foi de $17 \mathrm{~mm}$ e o máximo de $65 \mathrm{~mm}$, com uma maior frequência para a classe de 42 a $47 \mathrm{~mm}$ de comprimento $(\mathrm{n}=54)$ e o menor número de indivíduos para as classes de comprimento inferiores a $27 \mathrm{~mm}$.

A análise da distribuição por frequência de classe de comprimento nas quatro estações em estudo (Fig. 2), demonstra ausência de indivíduos menores que $17 \mathrm{~mm}$ na amostra, uma baixa frequência de exemplares com

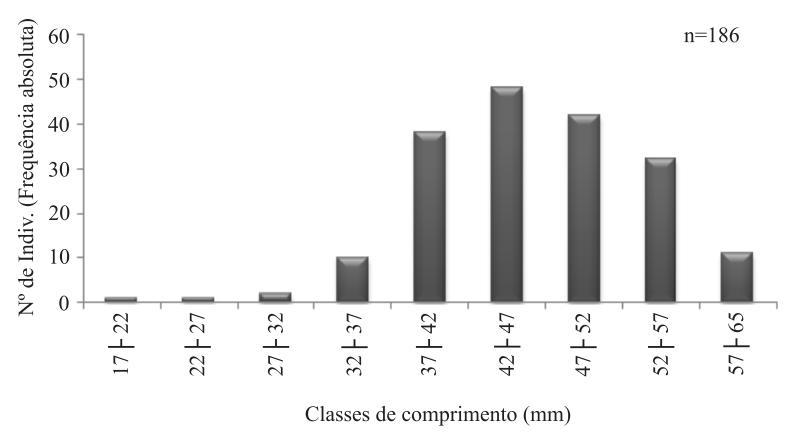

Figura 1. Distribuição por frequência de classes de comprimento (mm) de Diplodon expansus (Küster, 1856) no rio Piraquara, Paraná, Brasil, entre março e dezembro de 2006. 

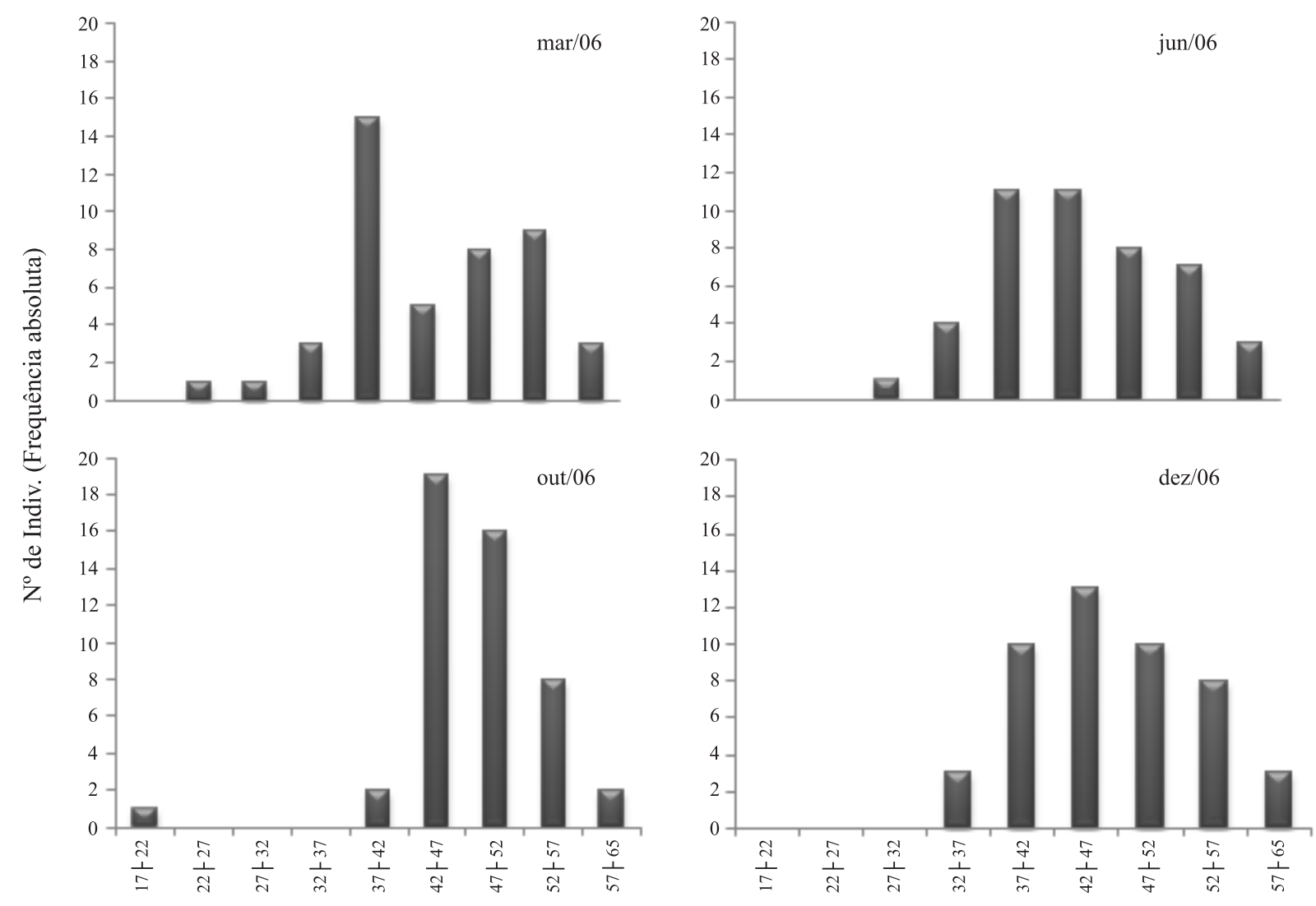

Frequência de classes de comprimento (mm)

Figura 2. Frequência de classes de comprimento (mm) por coleta amostral de Diplodon expansus (Küster, 1856) no rio Piraquara, Paraná, Brasil, entre março e dezembro de 2006.

comprimento entre 17 mm e 27 mm e uma maior frequência para exemplares de comprimento superior a $37 \mathrm{~mm}$. Nas quatro coletas realizadas, a classe modal está situada entre $37 \mathrm{~mm}$ e $52 \mathrm{~mm}$.

A determinação do sexo através de análise histológica permitiu a identificação de 94 machos e 92 fêmeas (Fig. 3). A análise da distribuição de frequência de machos e fêmeas ao longo de todo o período de estudo não evidenciou diferenças significativas, com a razão sexual observada de $1: 1 \quad(\mathrm{p}<0,05)$, não tendo sido verificado nenhum indivíduo hermafrodita.

As gônadas femininas (Fig. 4) e masculinas (Figs 8, 9) possuem estrutura arborescente, altamente ramificada e ocupam a maior porção da massa visceral, estando localizadas em compartimentos formados por septos musculares na região central e posterior.

Ao longo de todo período de coleta, as fêmeas apresentaram gônadas femininas contendo todos os estágios da ovogênese. Ovócitos imaturos com marcante basofilia citoplasmática, ovócitos em desenvolvimento presos ao epitélio germinativo através de pedúnculos citoplasmáticos e ovócitos maduros na luz do folículo (Figs 4, 5). As análises quantitativas demonstram uma diminuição no número e aumento no diâmetro de ovócitos na coleta de outubro, porém estas diferenças não são estatisticamente significativas (Figs 6, 7).

Em D. expansus os marsúpios estão localizados na porção média das brânquias internas. Fêmeas com
Tabela I. Parâmetros abióticos do rio Piraquara, Paraná, Brasil, obtidos nos períodos de coleta, entre março e dezembro de 2006.

\begin{tabular}{lcccc}
\hline & mar/06 & jun/06 & out/06 & dez/06 \\
\hline Temperatura da água & $18^{\circ} \mathrm{C}$ & $8^{\circ} \mathrm{C}$ & $11^{\circ} \mathrm{C}$ & $16^{\circ} \mathrm{C}$ \\
Condutividade $(20 \mu \mathrm{S} / \mathrm{cm})$ & 0,11 & 0,10 & 0,10 & 0,14 \\
Velocidade de corrente & $0,9 \mu / \mathrm{s}$ & $1,0 \mu / \mathrm{s}$ & $0,3 \mu / \mathrm{s}$ & $0,16 \mu / \mathrm{s}$ \\
\hline
\end{tabular}

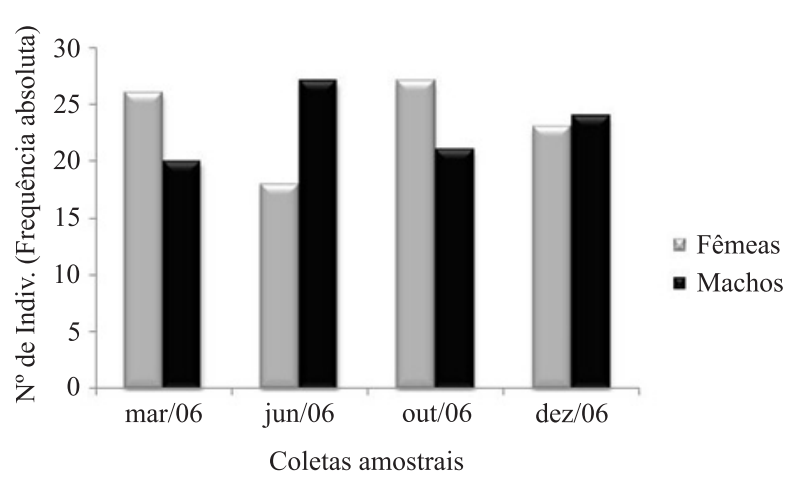

Figura 3. Frequência de machos e fêmeas, por coleta amostral, de Diplodon expansus (Küster, 1856) no rio Piraquara, Paraná, Brasil, entre março e dezembro de 2006.

marsúpios foram capturadas em todas as coletas amostrais e em todas as frequências de classe acima de $31 \mathrm{~mm}$ de comprimento. O maior número de fêmeas contendo marsúpios foi observado na coleta de outono $(80 \%)$ e o menor número na coleta de inverno (47\%). 


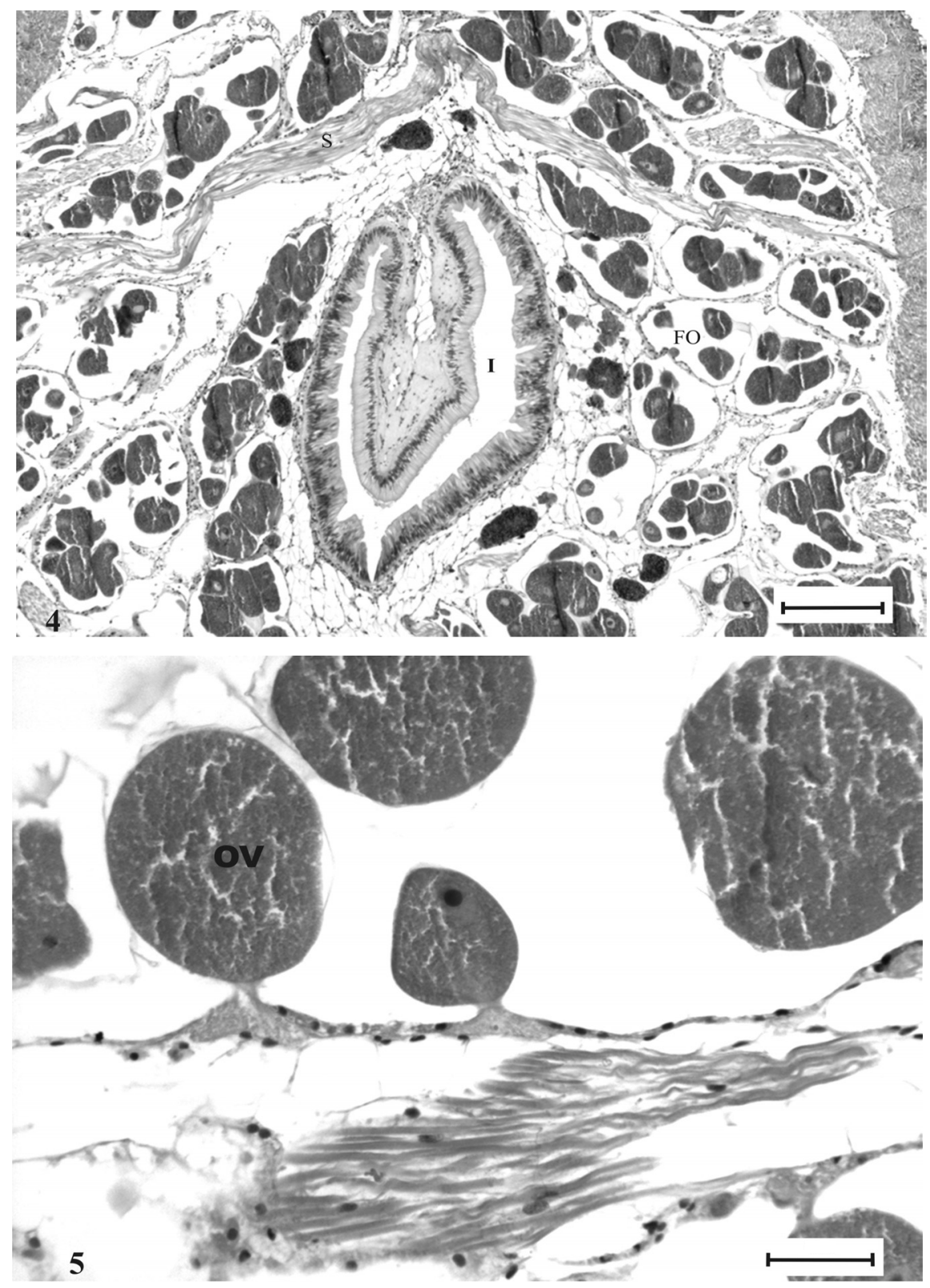

Figuras 4, 5. Aspectos histológicos das gônadas femininas de Diplodon expansus (Küster, 1856) capturados no rio Piraquara, Paraná, Brasil, entre março e dezembro de 2006 (I, intestino; S, septos musculares; FO, folículos gonadais com ovócitos maduros na luz folicular; OV, ovócitos em desenvolvimento). Escalas: Fig. 4, $250 \mu \mathrm{m}$; Fig. 5, $50 \mu \mathrm{m}$.

Nas gônadas masculinas, os folículos apresentam aglomerados de células localizados marcadamente em sua periferia (Figs 8, 9). Em todas as coletas foram observados diferentes estágios da espermatogênese identificados pela forma, tamanho e aspecto morfológico do núcleo. Espermatozóides flagelados foram observados na luz do folículo em todos os exemplares.

\section{DISCUSSÃO}

Segundo Alvarenga \& Ricci (1981) os Unionidea ocorrem em locais de fundo lodoso e MANSUR (1973) observou espécies de Diplodon em locais de areia fina. No rio Piraquara $D$. expansus encontra-se igualmente distribuído nas porções marginais, em que predomina substrato lodoso devido à mistura de silte com matéria orgânica proveniente da mata ciliar, e nas regiões centrais de fundo arenoso. HENRY \& SIMÃo (1984) não constataram relação significativa entre a granulometria do sedimento e a densidade de $D$. delontus expansus. Apesar do sedimento possuir grande importância para a distribuição de bivalves, diferentes fatores atuam de forma conjunta, como a presença de matéria orgânica em decomposição, características físicoquímicas da água e o caráter lótico ou lêntico do ambiente (Parada \& Peredo, 1994; Semenas \& Brugni, 2002). No rio Piraquara a concentração de exemplares é maior em áreas de baixa penetração de luz devido à presença de mata ciliar mais densa e menor concentração em locais de maior incidência luminosa, o que sugere que a luminosidade também possa influenciar a distribuição do $D$. expansus. 
6
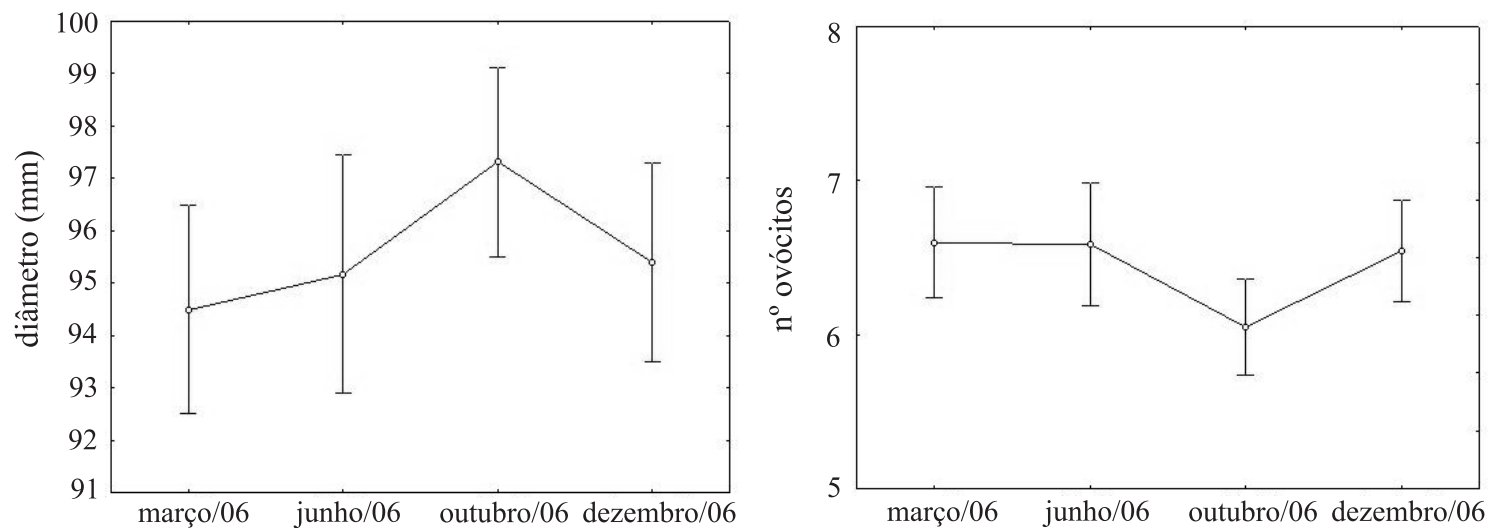

Figuras 6, 7. Ovócitos de Diplodon expansus (Küster, 1856) amostrados entre março e dezembro de 2006, no rio Piraquara, Paraná, Brasil: 6 , variação média do diâmetro de ovócitos; 7 , número de ovócitos.

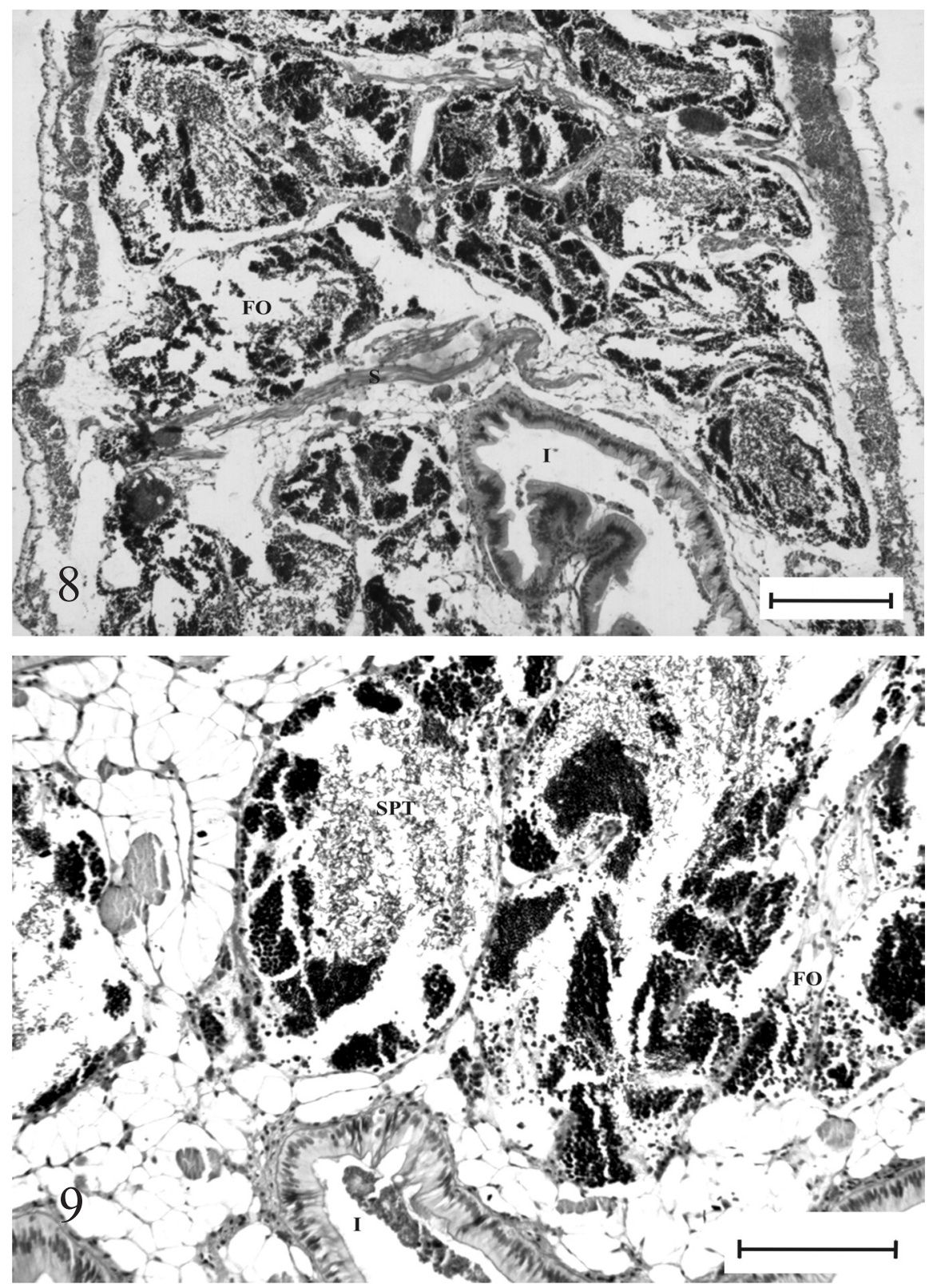

Figuras 8, 9. Aspectos histológicos das gônadas masculinas de Diplodon expansus (Küster, 1856) capturados no rio Piraquara, Paraná, Brasil, entre março e dezembro de 2006 (I, intestino; S, septos musculares; FO, folículos gonadais masculinos; SPT, espermatozóides). Escalas: Fig. 8, $300 \mu \mathrm{m}$; Fig. 9, $150 \mu \mathrm{m}$. 
A concentração de exemplares em uma determinada região, observada por LARA \& PARADA (1988) para $D$. chilensis e HENRY \& Simão (1984) para D. delontus expansus, tem sido atribuída à capacidade de selecionar substratos favoráveis, com maior disponibilidade de alimento (LARA \& Moreno, 1995) ou para aumentar o êxito reprodutivo da época de desova (Амуот \& Downing, 1988).

A análise das frequências de classes de comprimento da amostra $(n=186)$ demonstra que o maior número de indivíduos encontra-se nas classes intermediárias, o que está de acordo com o registrado por HENRY \& SIMÃo (1984) para D. delontus expansus no reservatório de Rio Pardo (SP). No entanto, o comprimento dos exemplares da classe modal e o tamanho dos maiores indivíduos no rio Piraquara é menor que o observado para o reservatório de Rio Pardo. Essas diferenças podem ser determinadas pelas diferenças físico-químicas dos ambientes ou por características interespecíficas.

O reduzido número de exemplares de pequeno porte e a ausência de exemplares de tamanho inferior a $17 \mathrm{~mm}$ na amostra, também registrada por HENRY \& SIMÃo (1984), sugere uma distribuição homogênea da população e ausência de recrutamento de juvenis no período estudado. Não se observa na amostra crescimento dos indivíduos, que seria caracterizado pelo deslocamento da classe modal, permitindo inferir que o período de crescimento da espécie é longo, uma característica dos moluscos bivalves (PARADA et al.,1989), e que o registro de diferenças significativas ocorre somente após um ano. Nas espécies de Hyriidae da América do Sul são descritos dois tipos de gloquídios, os que apresentam ciclo parasitário em peixes e os que apresentam desenvolvimento completo no marsúpio, até a fase de pós-larva (MANsur, 1999; MANsur \& Silva, 1999). Não existem registros na literatura sobre o tipo de gloquídio de D. expansus, no entanto é possível que a ausência de classes de tamanho inferiores ao observado possa ser atribuída à estratégia de dispersão da espécie cujas larvas aderidas ao hospedeiro possam ser liberadas em águas mais interiores (MANSUR, 1999) ou que os indivíduos na fase de pós-larva sejam transportados pela correnteza para outros locais do rio com substratos favoráveis ao desenvolvimento.

Na população estudada a proporção sexual de 1:1 e a ausência de hermafroditas demonstram que $D$. expansus no rio Piraquara é uma espécie tipicamente dióica, o que está de acordo com o observado para $D$. chilensis chilensis por PEREDO \& PARADA (1984). CURIAL \& LANGE (1974a, 1975) analisaram a proporção sexual de D. delontus expansus registrando a presença de hermafroditas na amostra; no entanto, segundo DUDGEON \& Morton, (1983), na família Hyriidae, o hermafrodistismo é eventual e exemplares hermafroditas ocorrem em baixa frequência em populações dióicas.

A estrutura arborescente e altamente ramificada das gônadas femininas e masculinas possuem o mesmo padrão observado por PEREDO \& PARADA (1984) para D. chilensis chilensis e por CURIAL \& LANGE (1975) para D. delontus expansus.
CuRial \& Lange (1975) compararam, através de análises qualitativas, indivíduos coletados no verão e inverno e observaram uma maior intensidade da gametogênese no período de inverno. No presente estudo, foram observadas todas as etapas da gametogênese feminina e masculina, nas quatro estações do ano, e a análise quantitativa demonstrou que as diferenças observadas não são significativas. Segundo HAGGERTY et al. (1995) os métodos quantitativos são mais indicados para análise da gametogênese porque resultam em um exame mais fiel da atividade gonadal e podem demonstrar variações intraespecíficas, além de possibilitarem comparações interespecíficas.

Os resultados do presente estudo sugerem que $D$. expansus no rio Piraquara apresenta um padrão de distribuição semelhante ao observado por outros autores. A população se apresentou como tipicamente dióica e a gametogênese contínua com desenvolvimento de gloquídios em todas as estações do ano indica um ciclo reprodutivo contínuo. O conjunto dos dados apresentados amplia o conhecimento sobre o gênero, possibilitando comparações intra- e interespecíficas com populações de outras localidades.

\section{REFERÊNCIAS BIBLIOGRÁFICAS}

Agostinho, A. A.; Thomas, S. M. \& Gomes, L. C. 2005. Conservação da Biodiversidade em águas continentais do Brasil. Megadiversidade 1(1):1-9.

Alvarenga, L. C. \& Ricci, C. N. 1981. Bivalvia. In: Hurlbert, S. H.; Rodrigues, G. \& Santos, N. D. eds. Aquatic biota of tropical south America. 2: Arthropoda. San Diego, San Diego State University. p.208-217.

Амyot, J. P. \& Downing, J. A. 1988. Locomotion in Elliptio complanata (Mollusca: Unionidae) a reproductive function. Freshwater Biology 39(2):51-358.

Avelar, W. E. P. \& Cunha, A. D. 2009. The anatomy and functional morphology of Diplodon rhombeus fontainianus (Orbigny, 1835) (Mollusca, Bivalvia, Hyriidae). Brazillian Journal Biology 69(4):1153-1163.

Avelar, W. E. P \& Mendonça, S. H. S. T. 1998. Aspects of gametogenesis of Diplodon rotundus gratus (Wagner, 1827) (Bivalvia: Hyriidae) in Brazil. American Malacological Bulletin 14(2):157-163.

Bonetto, A. 1961. Investigaciones acerca de las formas larvales em El género Diplodon y su aplicación a los estúdios sistemáticos. Santa $\mathrm{Fe}$, Dirección de Recursos Naturales, Ministério de Agricultura y Ganadería. 48p.

Curial, O. \& Lange, R. R. 1974a. Hermafroditismo em Diplodon delodontus expansus. Arquivos de Biologia e Tecnologia 17(2): 109-110.

1974b. Observações sobre a proporção de sexos em Diplodon delodontus expansus. Arquivos de Biologia e Tecnologia 17(2): 111,112

. 1975. Variações histológicas sasonais das gônadas do Diplodon delodontus expansus (Küster) (Mollusca, Unionidade). Arquivos de Biologia e Tecnologia 18(1):65-67.

Dudgeon, D. \& Morton, B. 1983. The population and sexual strategy of Anodonta woodiana (Bivalvia: Unionacea) in Plover Cove Reservoir, Hon Kong. Journal of Zoology 201:161-183.

Haggerty, T. M.; Garner, J. T.; Patterson, G. H. \& Jones, L. C., Jr. 1995. A quantitative assessment of the reproductive biology of Cyclonaias tuberculata (Bivalvia: Unionidae). Canadian Journal Zoology 73:83-88

Henry, R. \& Simão, C. A. 1984. Evalluation of density and biomass of a bivalve population (Diplodon delontus expansus) (Küster, 1856) in a small tropical reservoir. Revista Hydrobiologia Tropical 17(4):309-318. 
Jones, H. A.; Simpson, R. D. \& Humphrey, R. D. 1986. The reproductive cycles and glochidia of freshwater mussels (Bivalvia: Hyriidae) of the Macleay River, northern South Wales, Australia. Malacologia 27:185-2020.

Lara, G. \& Moreno, C. 1995. Efectos de la depredacíon de Egla abato (Crustacea, Aeglidae) sobre la distribución espacial y abundancia de Diplodon chilensis chilensis (Bivalvia, Hyriidae) en el Lago Panguipulli, Chile. Revista Chilena de Historia Natural 68:123-129.

Lara, G. \& Parada, E. 1988. Distribuicion espacial y densidad de Diplodon chilensis chilensis (Gray, 1828) en el Lago Villarrica $\left(39^{\circ} 18^{\prime} \mathrm{S}\right.$; $\left.72^{\circ} 05^{\prime} \mathrm{W}\right)$. Boletin de La Sociedad de Biologia de Concepcion 59:105-114.

MaAck, R.1981. Geografia física do Estado do Paraná. 2. ed., Rio de Janeiro, Olympio. 442 p.

Mansur, M. C. D. 1973. Morfologia do sistema digestivo das espécies do gênero Diplodon Spix, 1827 do rio Guaíba, Rio Grande do Sul (Unionacea - Hyriidae). Iheringia, sér. Zool., 43:75-90.

1999. Gloquídio de Diplodon martensi (Ihering) (Mollusca, Bivalvia, Hyriidae) e seu ciclo parasitário. Revista Brasileira de Zoologia 16(2):185-194.

Mansur, M. C. D. \& Pereira, D. 2006. Bivalves límnicos da bacia do rio dos Sinos, Rio Grande do Sul, Brasil (Bivalvia, Unionoida, Veneroida e Mytiloida). Revista Brasileira de Zoologia 23(4):1123-1147.

Mansur, M. C. D.; Schulz, C. \& Garces, L. M. M. P. 1987. Moluscos Bivalves de água doce: identificação dos gêneros do sul e leste do Brasil. Acta Biologica Leopoldensia, 9(2):181-202.

Mansur, M. C. D. \& Silva, M. G. O.1999. Description of glochidia of five species of freshwater mussels (Hyriidae: Unionoidea) from South América. Malacologia 41(2):475-483.

Marques, P. H. C.; Oliveira, H. T. \& Machado, E. C. 2003. Limnological study of Piraquara river (Upper Iguaçu Basin): spatiotemporal variation of physical and chemical variables and watershed zoning. Brazilian Archives of Biology and Tecnology 46(3):-394.

MMA (Ministério do Meio Ambiente). 2008. Livro Vermelho das espécies da fauna ameaçada de extinção. Disponível em: < w w w.mma.gov.br/estruturas/179/arquivos/ vol_i_invertebrados_aquaticos.pdf >. Acesso em: 10.07.2010

Parada, E. \& Peredo, S. 1994. Un enfoque ecológico evolutivo de lãs estratégias de historia de vida de los híridos chilenos (Mollusca: Bivalvia). Boletin de La Sociedad de Biologia de Concepcion 65:71-80.

Parada, E.; Peredo, S. \& Gallardo, C. 1990. Tácticas reproductivas y dinámica poblacional de Diplodon chilensis (Gray, 1828) Bivalvia: Hyriidae). Revista Chilena de Historia Natural 63:23-35.

Parada, E.; Peredo, S.; Lara, G. \& Valdebenito, I. 1989. Growth, age and life span of the freshwater mussel Diplodon chilensis chilensis (Gray, 1828). Archives Hydrobiologie 115(4):563-573.

Parada, E. Peredo, S.; Valenzuela, J. \& Manuschevich, D. 2007. Extention of the current northern distribution range of freshwater mussel Diplodon chilensis (Gray, 1828) (Bivalvia: Hyriidae) in Chile. Gayana 71(2):212-215.

Parodiz, J. J. \& Bonetro, A. A. 1963. Taxonomy and zoogeographic relationsships of the South American naiades (Bivalvia: Unionacea and Mutelacea). Malacologia 1:179-213.

Peredo, S. \& Parada, E. 1984. Gonadal organization and gametogenesis in the fresh-water mussel Diplodon chilensis chilensis (Mollusca: Bivalvia). The Veliger 27(2):126-133.

Semenas, L. \& Brugni, N. 2002. Características poblacionales y ciclo de vida de Diplodon chlilensis (d'Orbigny, 1835) (Hyriidae, Bivalvia) en el Lago Gutiérrez (Patagonia, Argentina). Ecologia Austral 12:29-40.

Vaughn, C. C. \& Hakenkamp, C. C. 2001. The Functional role of burrowing bivalves in freshwater ecosystems. Freshwater Biology 46:1431-1446.

Recebido em agosto de 2009. Aceito em dezembro de 2010. ISSN 0073-4721

Artigo disponível em: www.scielo.br/isz

Impresso e distribuído em 2011. 